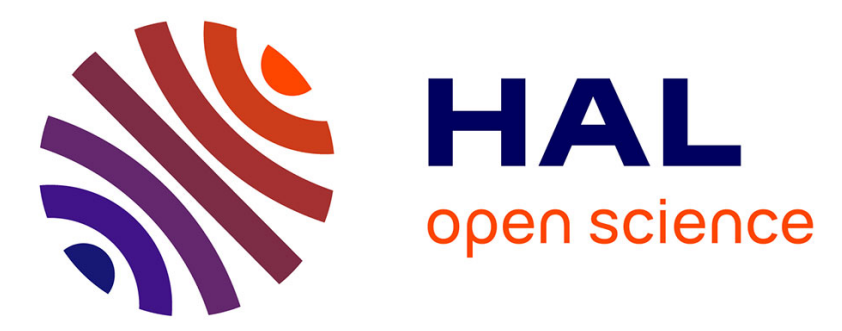

\title{
Long-term persistence of the spatial organization of temperature fluctuation lifetime in turbulent air avalanches
}

\author{
C Crouzeix, J.-L Le Mouël, F Perrier, M. G Shnirman, E Blanter
}

\section{- To cite this version:}

C Crouzeix, J.-L Le Mouël, F Perrier, M. G Shnirman, E Blanter. Long-term persistence of the spatial organization of temperature fluctuation lifetime in turbulent air avalanches. Physical Review E : Statistical, Nonlinear, and Soft Matter Physics, 2006, 74 (3), pp.036308. 10.1103/PhysRevE.74.036308 . insu-01295492

\section{HAL Id: insu-01295492 \\ https://hal-insu.archives-ouvertes.fr/insu-01295492}

Submitted on 31 Mar 2016

HAL is a multi-disciplinary open access archive for the deposit and dissemination of scientific research documents, whether they are published or not. The documents may come from teaching and research institutions in France or abroad, or from public or private research centers.
L'archive ouverte pluridisciplinaire HAL, est destinée au dépôt et à la diffusion de documents scientifiques de niveau recherche, publiés ou non, émanant des établissements d'enseignement et de recherche français ou étrangers, des laboratoires publics ou privés. 


\title{
Long-term persistence of the spatial organization of temperature fluctuation lifetime in turbulent air avalanches
}

\author{
C. Crouzeix, ${ }^{1}$ J.-L. Le Mouël, ${ }^{1}$ F. Perrier,${ }^{1}$ M. G. Shnirman,,${ }^{1,2}$ and E. Blanter ${ }^{1,2}$ \\ ${ }^{1}$ Équipe de géomagnétisme, Institut de physique du Globe, Boîte Postale 89, 4 place Jussieu, 75252 Paris cedex 05, France \\ ${ }^{2}$ International Institute for Earthquake Prediction Theory and Mathematical Geophysics, Warshavskoe shosse, 79, korp2, \\ Moscow 113556, Russia
}

(Received 19 September 2005; published 27 September 2006)

\begin{abstract}
It has been recently proposed that some natural phenomena, such as sunspot occurrence, can be represented by a modulated Markov jitter, which is a high-frequency Markov signal multiplied by a long-term component. The two parameters of this model can be estimated using a nonlinear method based on absolute derivatives. This analysis is applied here to a different physical system: the temperature time series measured during air avalanches in the vertical access pit of an underground quarry. The thermal fluctuations associated with these turbulent flows, driven by the external temperature forcing, actually appear as another practical realization of a modulated Markov jitter. One parameter of the model provides the lifetime of the temperature fluctuations, which can be estimated as a function of time and position. The obtained lifetime is of the order of 10 to $25 \mathrm{~min}$, and is remarkably constant in time for each sensor, independently of the amplitude of the forcing. Furthermore, a significant and persistent spatial structure is observed, revealing a long-term intrinsic organization of the turbulent air flows in the pit. Such a stable spatial organization may reflect a general feature of turbulent phenomena.
\end{abstract}

DOI: 10.1103/PhysRevE.74.036308

PACS number(s): 47.27.E-

\section{INTRODUCTION}

Nonequilibrium turbulent systems are a fascinating area of research and, in particular, they exhibit an amazing ability to organize themselves into persistent structures [1]. The origin of this spatial organization and its emergence in noisy or chaotic environments are poorly understood at the moment. This organization is mainly investigated in well-controlled Rayleigh-Bénard experiments where, in the hard-turbulence regime, the onset of large-scale circulation appears connected to the statistical properties of plumes [2,3]. Such phenomena are not specific to particular laboratory experiments, however, but seem to be universal, pervading natural processes at all scales [4].

It is interesting to develop simple representations with few parameters whose spatial and temporal variations can be studied easily. One representation has demonstrated a definite relevance in the case of sunspots [5]: a high-frequency Markov process [6] multiplied by a long-term component, the modulated Markov jitter. Using a simple nonlinear technique based on the absolute derivative, one parameter of the signal, its lifetime, has been determined and, in the case of sunspots, observed to increase with time [7]. This leads us to search for other realizations of the modulated Markov jitter in different natural contexts.

In a previous paper [8], we analyzed the properties of turbulent air avalanches in the 20-m-deep vertical access pit to an underground cavity. The aim of this paper was to describe the properties of turbulent flows in a medium-scale natural system and discuss it in the light of Rayleigh-Bénard (RB) convection and general turbulence, e.g., [9]. Obviously, this system differs from the standard RB experiments, in particular because of its boundary conditions: at the top and at the bottom of the pit, exchanges take place with the outside and with the quarry; heat transfers also occur at the walls. This system is thus open and nonadiabatic.

Air avalanches take place in the pit when the outside air density is larger than the equilibrium air density in the quarry, thus most of the time in winter, when the outside air is colder than the quarry air. During this regime, the mean Rayleigh number $\mathrm{Ra}$ is estimated to be about $10^{12}$, and the Prandtl number Pr of air is about 0.7. Temperature measurements performed along a vertical profile show that the avalanches are associated with a mean vertical temperature profile and with broadband, spatially organized, and coherent temperature fluctuations [8]. This analysis has shown that persistent structures of the flow can be observed, apparently imposed by the boundary conditions.

In the present paper, we are not investigating further the underlying fluid dynamics of the air avalanches in the framework of standard RB convection. Rather, we study the relevance of a modulated Markov jitter to describe the temperature time series observed in the pit. As developed in the case of sunspots, we apply our method of absolute derivatives and we study the spatial and temporal variations of the estimated parameters, independently of any a priori assumption on the nature of the turbulent flows.

We first recall the properties of the modulated Markov jitter, and we briefly describe the site and the experimental setup and state the results obtained in previous studies. The method of absolute derivatives is then applied to the temperature time series and we study the estimated lifetime as a function of time and position. The relevance of the modulated Markov jitter is discussed in the conclusion in relation with sunspots.

\section{MODULATED MARKOV JITTER}

Modulated Markov jitter was proposed to represent the sunspot activity by Blanter et al. [5]. We succinctly recall its 
properties here. The signal $\Theta(t)$, taken as a positive real number, is written as

$$
\Theta(t)=\eta(t) F(t)
$$

i.e., the product of a long-term (low-frequency) component $F$ and a high-frequency component $\eta$, referred to as the jitter. The conditions of validity of the representation (1) are discussed by Blanter et al. [5]. Grossly speaking, it is valid if the $\Theta\left(t_{i}\right)$ time series is similar to the time series made of its discrete absolute derivative, defined as

$$
\left|\Theta^{\prime}\left(t_{i}\right)\right|=\frac{\left|\Theta\left(t_{i+1}\right)-\Theta\left(t_{i}\right)\right|}{\delta t},
$$

where $\delta t=t_{i+1}-t_{i}$ is the sampling interval. In practice, the choice of such a simple model is justified by the results. Let us point out that absolute values of turbulence parameters (velocity and temperature increment) have already been used, although in a different way $[10,11]$. Taking absolute values enhances the effect of positive and negative fluctuations, while they are canceled when simple moving averages are computed.

Here, we further assume that the jitter has the following form:

$$
\eta\left(t_{i+1}\right)=\alpha \eta\left(t_{i}\right)+\xi\left(t_{i+1}\right) .
$$

Equation (3) is the general form of an autoregressive Markov process of first order; the first term of the right-hand side is what remains at time $t_{i+1}$ of the value of the process at time $t_{i}$, whereas the second term $\xi$ is a newly generated flow. The autoregression coefficient $\alpha$ characterizes the correlation between the two successive variables $\eta\left(t_{i+1}\right)$ and $\eta\left(t_{i}\right)$. In Blanter et al. [5], the random variable $\xi$ is arbitrarily taken as uniformly distributed on the interval $(0,1), \xi \in \cup[0,1]$. In the present paper, we rather consider a binomial variable $\xi$ $=B(p) ; \xi=1$ with probability $p$, and $\xi=0$ with probability $(1-p)$.

We assume, for the sake of simplicity, the low-frequency modulation $F$ positive and sinusoidal with a period $T$. Here, this represents the daily modulation of the pit temperature due to the daily external forcing. In order to investigate the properties of the high-frequency component $\eta$, we have to eliminate the influence of the modulation $F$. Therefore, most of the time, we will average $\Theta\left(t_{i}\right)$ over time intervals of length $T$ using

$$
\Theta_{T}\left(t_{i}\right)=\frac{1}{N} \sum_{k=i-n}^{i+n} \Theta\left(t_{k}\right),
$$

with $T=N \delta t$ and $N=2 n+1$. The mean lifetime $\tau$ can be written [5]

$$
\tau=\frac{\delta t}{1-\alpha}
$$

To estimate $\tau$ from $\Theta\left(t_{i}\right)$, we introduce the inverse relative variation (IRV) of the signal as the ratio of the mean value of the signal to the mean value of its absolute derivative:

$$
r_{T}(t)=\frac{\Theta_{T}(t)}{\left|\Theta^{\prime}\right|_{T}(t)} .
$$

The average value over $T$ of the modulated Markov jitter defined by Eqs. (1) and (3), with $\xi \in B(p)$, is approximately

$$
\Theta_{T}(t)=\frac{p F_{T}(t)}{1-\alpha},
$$

and the average of its absolute derivative is

$$
\left|\Theta^{\prime}\right|_{T}(t)=\frac{2 p(1-p) F_{T}(t)}{\delta t} .
$$

The IRV thus does not depend on the modulation $F$, but only reflects the properties of the high-frequency component:

$$
r_{T}(\Theta)=r=\frac{\delta t}{2(1-p)(1-\alpha)} .
$$

The Parameter $p$ is also estimated from the data; a practical estimator is the relative frequency of positive values of $\Theta^{\prime}(t)$ :

$$
\hat{p}=P\left\{\Theta\left(t_{i+1}\right)-\Theta\left(t_{i}\right)>0\right\} .
$$

The estimate of the lifetime $\hat{\tau}_{r}$ through $r$ is given by

$$
\hat{\tau}_{r}=2(1-\hat{p}) r
$$

\section{THE SITE AND THE EXPERIMENTAL SET-UP}

The abandoned "La Brasserie" limestone quarry is located $18 \mathrm{~m}$ below ground surface under the park of Vincennes near Paris [12]. It consists of pillars and rooms with a height varying from 2 to $7 \mathrm{~m}$, and a total air volume of approximately $60000 \mathrm{~m}^{3}$. The quarry is connected to the ground surface by a single large access pit (Fig. 1) with $4.56 \mathrm{~m}$ diameter. This pit of height $h=20 \mathrm{~m}$ can thus be considered as a vertical pipe linking the outside atmosphere, where temperature and relative humidity $(\mathrm{RH})$ vary on daily and seasonal cycles, to a finite but large volume of air at $12.7^{\circ} \mathrm{C}$ and $100 \%$ RH. A metallic staircase of diameter about $2.2 \mathrm{~m}$ slightly obstructs the air flow, leaving a free area of $15.4 \mathrm{~m}^{2}$ in the pit. The upper part of the pit reaches into a building, but broken panes in windows with a surface area of about $2.3 \mathrm{~m}^{2}$ allow air exchange with the outside atmosphere.

Half of the year, as long as the outside air density is larger than the equilibrium density of the quarry air, avalanches take place in the pit [13], producing natural ventilation of the whole quarry. The presence of this natural ventilation is confirmed by the seasonal variation of the radon concentration [14], which indicates ventilation rates varying from 0.03 to $0.14 \mathrm{~m}^{3} \mathrm{~s}^{-1}$. The air temperature in the quarry has an average value of $\theta_{0}=12.7^{\circ} \mathrm{C}$ with yearly variations of the order of $0.16^{\circ} \mathrm{C}$. The relative humidity is larger than $99.8 \%$ in nonventilated conditions, and varies from $99.2 \%$ to $99.8 \%$ during ventilated conditions in winter.

Temperature measurements in the pit are performed with two setups [8]. The first one is a vertical profile located about $65 \mathrm{~cm}$ away from the staircase, and consisting in ten points 


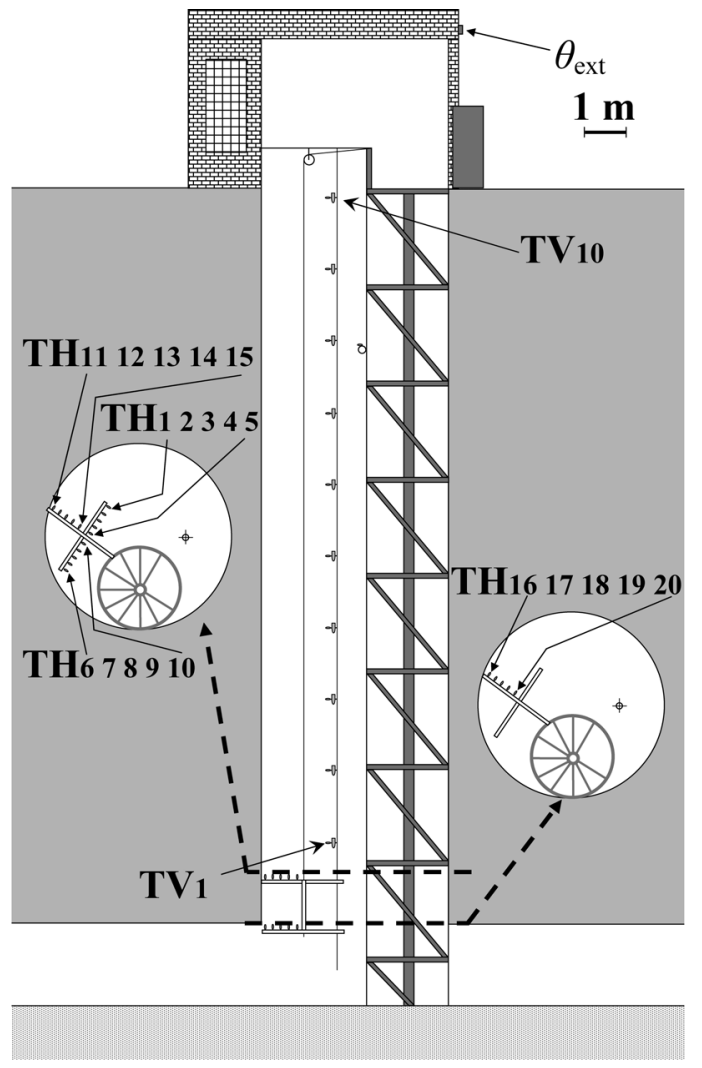

FIG. 1. Sketch of the access pit showing the vertical and the horizontal setups, TV and TH, respectively, and the location of the sensor measuring the outside temperature $\theta_{\text {ext }}$.

separated by $1.75 \mathrm{~m}$, labeled TV1-TV10 (Fig. 1). Because of their finite size, the thermistors have a response time of the order of $30 \mathrm{~s}$. The second setup consists in four horizontal branches of five thermistors each, with a regular spacing of $18 \mathrm{~cm}$. Three branches are located at the same level, and the fourth branch $1.3 \mathrm{~m}$ below, as indicated in Fig. 1. The sampling time interval is $2 \mathrm{~min}$. The outside temperature is recorded above the entrance door of the quarry pit building, with $1 \mathrm{~h}$ sampling interval.

\section{WINTER TEMPERATURE RECORDINGS; PREVIOUS RESULTS}

Temperatures $\theta$ recorded outside and along the vertical profile during four days of December 2003 are shown in Fig. 2(a). Note that the temperature in the upper part of the pit (TV9) is almost the average between the outside temperature $\theta_{\text {ext }}$ and the equilibrium temperature of the quarry $\theta_{0}$; this fact indicates a vigorous mixing of the air in the pit during the avalanches. Figure 2(b) presents the temperature $\theta$ averaged using Eq. (4) with $T$ equal to one day. A linear vertical profile of temperature is observed, which reflects heat exchanges at the wall $[8,13]$. The slope of this profile depends on the forcing.

During the winter regime, short-period fluctuations of the order of $1{ }^{\circ} \mathrm{C}$ peak to peak, which reflect the air avalanches, are noticed on all the sensors of the vertical profile. Distri-

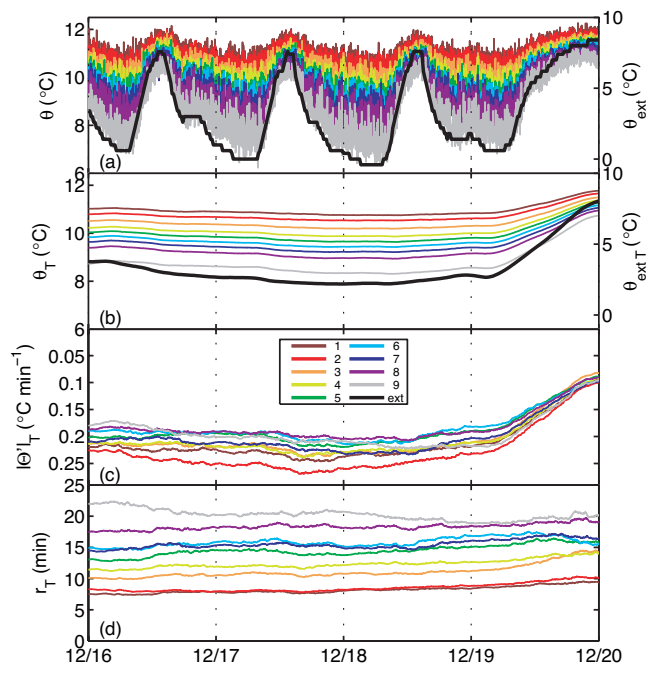

FIG. 2. (Color) Data recorded by the vertical set-up during four days of December 2003; (a) shows the raw data, i.e. temperature measured outside $\left(\theta_{\text {ext }}\right)$, and by sensors 1 to 9 of the set-up; (b) corresponds to the moving average calculated with Eq. (4) and $T$ equal to one day; (c) is the absolute derivative $\left|\Theta^{\prime}\right|_{T}$ as described by Eqs. (2) and (4); and (d) is the ratio $r_{T}$ (Eq. (6)).

butions of these temperature fluctuations are nearly Gaussian and their power density spectra are almost constant in the range from $3 \times 10^{-4}$ to $4 \times 10^{-3} \mathrm{~Hz}$, also a consequence of the heat transfers occurring at the wall [8]. These temperature fluctuations, which are coherent from sensor to sensor, have a standard deviation proportional to the temperature difference between the upper part of the pit and the equilibrium temperature of the quarry [13]. This property leads us to propose that the temperature fluctuations, from a statistical point of view, can be represented by a modulated Markov jitter.

\section{APPLICATION TO THE PIT TEMPERATURE TIME SERIES}

In the following, we define the signal as

$$
\Theta(t)=\theta_{0}-\theta(t)
$$

with $\theta_{0}=12.7^{\circ} \mathrm{C}$ the mean temperature in the quarry during December 2003. This temperature $\theta_{0}$ can be determined from the data themselves. Indeed, in Fig. 3, $\left|\Theta^{\prime}\right|_{T}$ is shown as a function of $\Theta_{T}$ for the various sensors of the vertical setup. $\left|\Theta^{\prime}\right|_{T}$ varies linearly with $\Theta_{T}$. All lines cross $\left|\Theta^{\prime}\right|_{T}=0$ almost at the same point; this value is determined to be $\theta_{0} \simeq 12.7^{\circ} \mathrm{C}$.

We consider first the data of December 2003 and January 2004 during which the outside temperature remained lower than the equilibrium temperature of the quarry, maintaining a winter regime. We compute $\Theta_{T}(t)$ and $\left|\Theta^{\prime}\right|_{T}(t)$ using Eqs. (4) and (2), with $T$ equal to one day.

Figure 4(a) displays the curves $\Theta_{T}(t)$ for sensors 1 to 9 of the vertical setup (see Fig. 1). It is quite remarkable how the nine curves are proportional to each other, even in their tiny features. Figure 4(b) displays the nine corresponding curves 


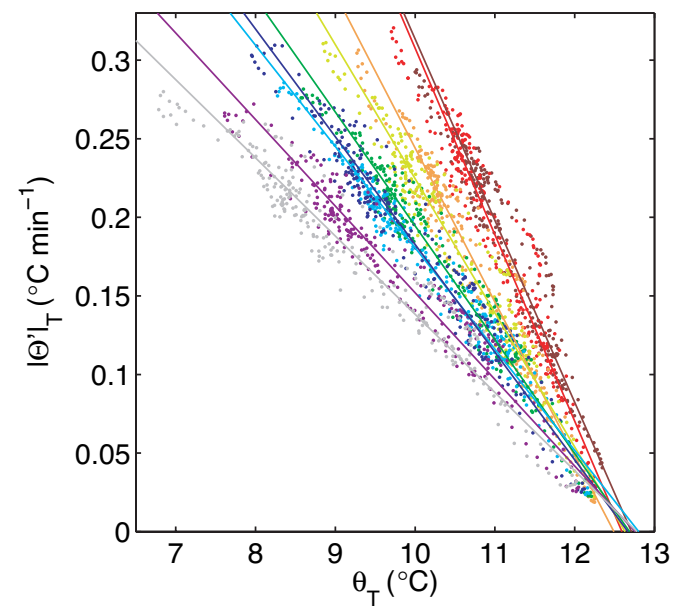

FIG. 3. (Color) Absolute derivative $\left|\Theta^{\prime}\right|_{T}$ as a function of mean temperature $\theta_{T}$ for sensors 1-9 of the vertical setup during December 2003. The linear trend (black line) calculated for each sensor is represented to estimate $\theta_{0}=12.7^{\circ} \mathrm{C}$. The color code is the same as for Fig. 2.

$\left|\Theta^{\prime}\right|_{T}(t)$. Again the $\left|\Theta^{\prime}\right|_{T}$ curves are strikingly similar, although a little less similar than are the $\Theta_{T}$ curves. Furthermore, the $\Theta_{T}$ curves are similar to the $\left|\Theta^{\prime}\right|_{T}$ curves, as already indicated by Fig. 3. This is the first condition for considering a modulated Markov jitter, as described by Blanter et al. [5]. The amplitude of the long-term variations of $\Theta_{T}(z, t)$ decreases when $z$, counted greater than 0 downward, increases, whereas the amplitude of the corresponding variations of $\left|\Theta^{\prime}\right|_{T}(z, t)$ is remarkably constant when $z$ varies, although to a lesser degree for the two uppermost sensors (see also [8] and [13]).

Figures 5(a) and 5(b) display the curves $\Theta_{T}(t)$ and $\left|\Theta^{\prime}\right|_{T}(t)$, respectively, calculated with the data of the

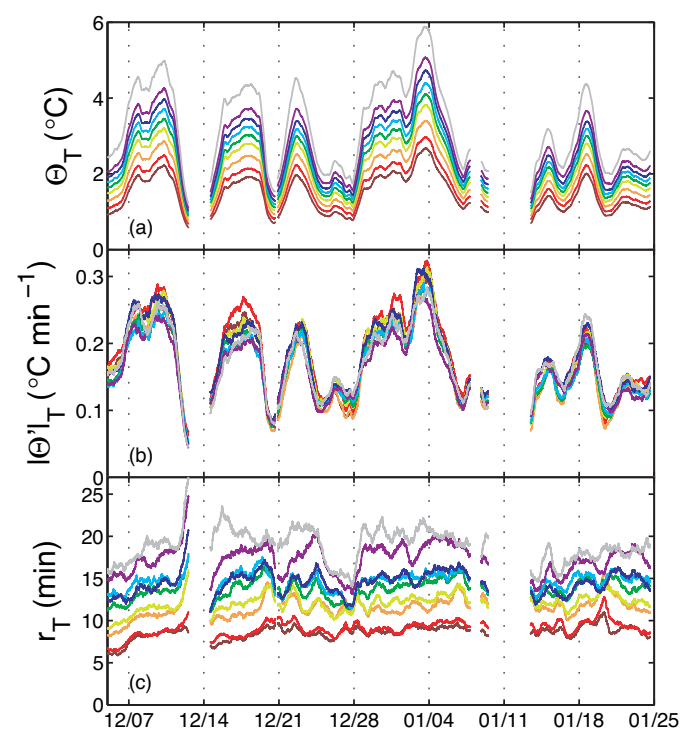

FIG. 4. (Color) Data recorded by the vertical setup during December 2003 and January 2004; (a) shows the moving average $\Theta_{T}$ calculated with Eq. (4) and $T$ equal to one day; (b) is the absolute derivative $\left|\Theta^{\prime}\right|_{T}$ as described by Eq. (2); and (c) is the ratio $r_{T}$ [Eq. (6)]. The color code is the same as for Fig. 2.

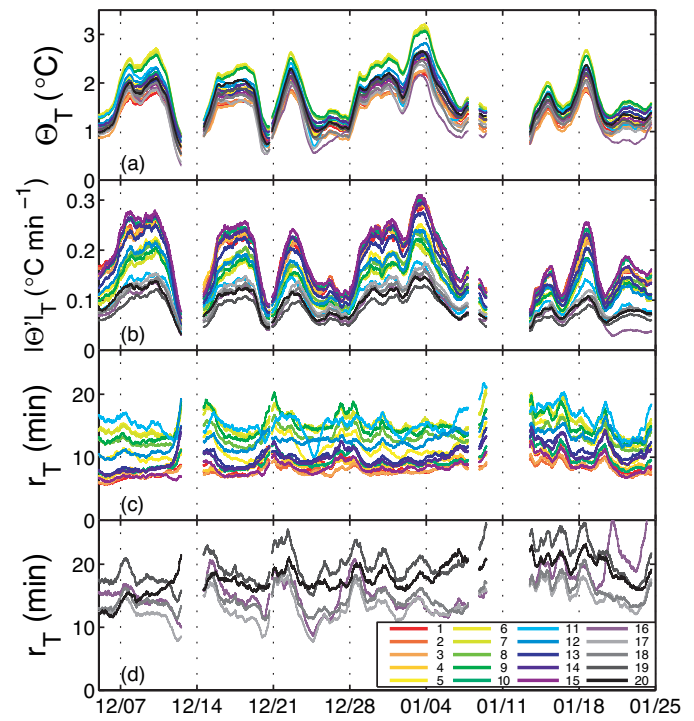

FIG. 5. (Color) Data recorded by the horizontal setup during December 2003 and January 2004; (a) shows the moving average $\Theta_{T}$ calculated with Eq. (4) and $T$ equal to one day; (b) is the absolute derivative $\left|\Theta^{\prime}\right|_{T}$ as described by Eqs. (2) and (4); and (c) and (d) represent the ratio $r_{T}$ [Eq. (6)] for sensors 1-15 and 16-20, respectively.

horizontal setup during the same period as for Fig. 4. By contrast with the vertical setup, $\Theta_{T}(t)$ is now almost constant horizontally, while $\left|\Theta^{\prime}\right|_{T}(t)$ varies from one sensor to another. However, $\Theta_{T}(t)$ and $\left|\Theta^{\prime}\right|_{T}(t)$ keep their similarity in time.

According to Eq. (6), we compute the ratio $r_{T}\left(t_{k}, \mathbf{x}_{i}\right)$ for each sensor of the two setups, at each time $t_{k}$ and each position $\mathbf{x}_{i}$ [Figs. 4(c), 5(c), and 5(d)]. The ratio $r_{T}\left(t_{k}\right)$ is fairly constant with time at each depth for the seven lower sensors [Fig. 4(c)], and for most of the sensors of the horizontal profile [Fig. 5(c)]. Larger irregularities, with time constants of a few days, are present on the curves relative to sensors 8 and 9 of the vertical setup, and to sensors 5, 11, and 16-20 of the horizontal setup.

Finally, to get a global description of the spatial variations of the properties of the signal, we compute the average value $\Theta_{T}$ and $\left|\Theta^{\prime}\right|_{T}$ of $\Theta\left(t_{k}, z_{i}\right)$ and $\left|\Theta^{\prime}\right|\left(t_{k}, z_{i}\right)$ from the vertical profile over four periods of ten days each, in December 2003, January 2004, December 2004, and January 2005. The distributions with depth of $\Theta_{T}$ and $\left|\Theta^{\prime}\right|_{T}$ are reported in Fig. 6 . The vertical profiles of $\Theta_{T}$ are almost linear with depth, as previously observed [8]. The slope with $z$ varies with time, and increases with the forcing, i.e., when the outside temperature decreases. Vertical variations of $\left|\Theta^{\prime}\right|_{T}$ are weak. Like $\Theta_{T},\left|\Theta^{\prime}\right|_{T}$ increases with the forcing, but its variation with $z$ remains identical.

Figure 7 represents the vertical profiles of the corresponding time averaged ratio $r$, computed over the same periods. As expected, in a first approximation, the shape of the profile is linear with depth. Its variations with time are small, and unrelated to the forcing.

The time-averaged ratio $r$, computed during December 2003 and January 2004 over two periods of ten days each, is shown in Fig. 8 versus the distance between the sensor and 


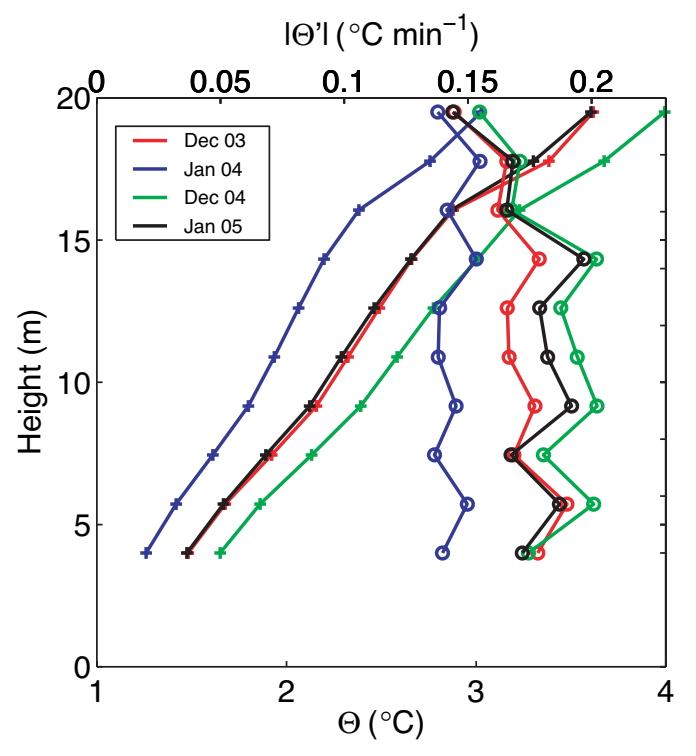

FIG. 6. (Color) Mean vertical profiles of $\Theta(+)$ and $\left|\Theta^{\prime}\right|(\circ)$, each computed over ten days during December 2003, January 2004, December 2004, and January 2005.

the wall of the pit. For sensors 1 to 15 (plain lines), $r$ decreases with horizontal distance. For sensors 16 to 20 (dotted lines), which are placed lower (see Fig. 1) and are actually located more in the quarry air than in the pit, $r$ increases with distance to the wall.

To summarize, in the case of the vertical temperature profile, the amplitude of $\Theta_{T}$ varies with position, while $\left|\Theta^{\prime}\right|_{T}$ remains constant. In the case of the horizontal profile, it is the opposite: $\left|\Theta^{\prime}\right|_{T}$ varies with position, while $\Theta_{T}$ remains constant. The vertical spatial variation of the IRV is thus due to $\Theta_{T}$ whereas its horizontal variations is due to $\left|\Theta^{\prime}\right|_{T}$. In both cases, the obtained IRV is stable with time.

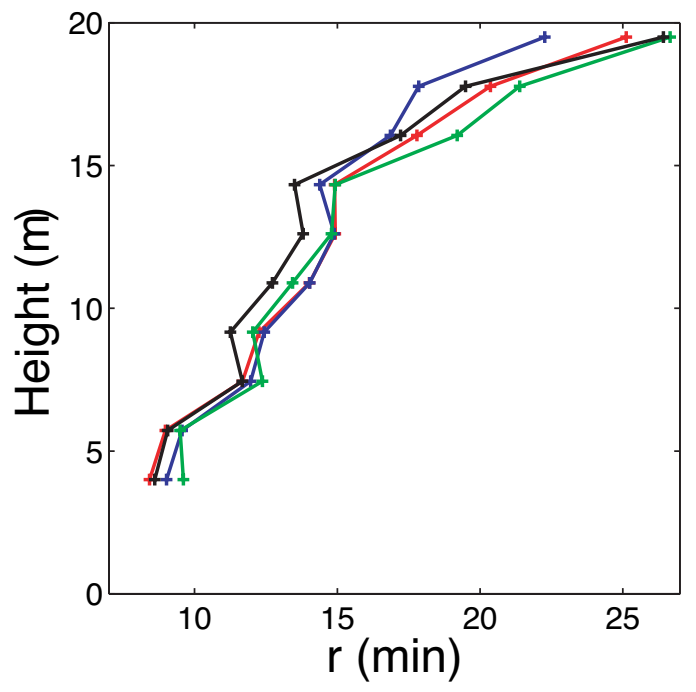

FIG. 7. (Color) Mean vertical profiles of $r$, each computed over ten days during December 2003, January 2004, December 2004, and January 2005. The color code is the same as for Fig. 6.

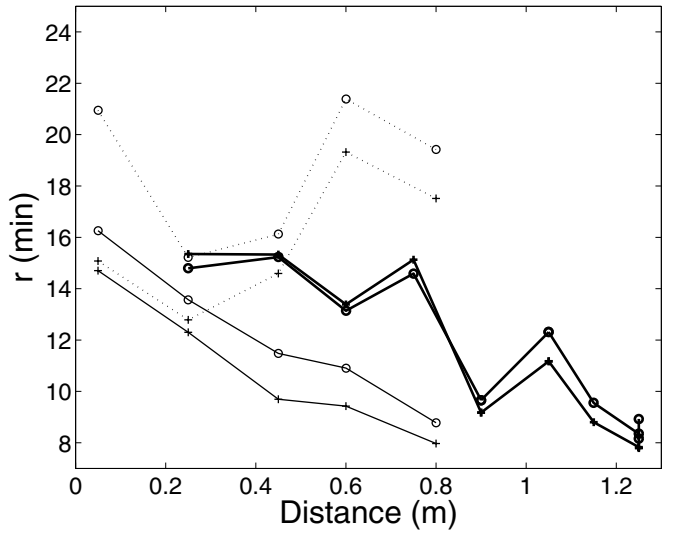

FIG. 8. Mean horizontal profiles of $r$ as a function of the distance from the wall, each computed over ten days during December 2003 (+), and January 2004 ( ${ }^{\circ}$. Bold lines correspond to sensors $1-10$, normal lines to sensors $11-15$, and dotted lines to sensors $16-20$.

\section{DETERMINATION OF LIFETIME AND DISCUSSION}

Having observed that the model of modulated Markov jitter can be used to describe the cold avalanches in the quarry pit, we can now determine the values of the two parameters of this model: $p$ and $\tau$. The value of $\hat{p}$, obtained from Eq. (10), is $0.50 \pm 0.01$, stable with time. No dependence versus position is observed.

The lifetime $\tau$ is the most interesting parameter because it bears a direct physical interpretation. It represents the mean duration of the cold avalanches at a given point. For a sampling time of 2 min, using Eq. (11), we obtain values varying from $8 \mathrm{~min}$ for sensor 1 to $14 \mathrm{~min}$ for sensor 7 (Fig. 7). For the horizontal profiles, considering sensors 1-15 (full lines in Fig. 8), the obtained value is about 15 min near the wall, decreasing to $8 \mathrm{~min}$ at $0.8-1.2 \mathrm{~m}$ from the wall. These values are significantly larger than the response time of the sensors (10-30 s, see Sec. III). When data with a shorter sampling time are used, for example $10 \mathrm{~s}$, the inferred values using Eq. (11) are three times smaller; however, $\hat{p}$ does not change.

The fact that the lifetime does not depend on time and thus on the forcing, which, equivalently, means that the avalanches are adequately represented by a simple modulated Markov jitter, is nontrivial. Also, the fact that the spatial structure of the lifetime persists with time provides a significant additional information. The flow, organized in a stable manner, has an intrinsic dynamics, independent of the forcing. Despite the inherently turbulent nature of the air avalanches, a spatial coherence is remarkably maintained in the system, maybe by the heat exchange at the wall. Thus, contrary to expectations, the cold avalanches do not mix and wash away spatial structures in the pit, but create and maintain their own. Certainly a robust and efficient mechanism is needed, but its mode of operation remains unclear. Such mechanisms seem to emerge in various types of natural systems at all scales $[1,4]$.

The modulated Markov jitter was noted to be also a good representation of the sunspot Wolf number as a function of 


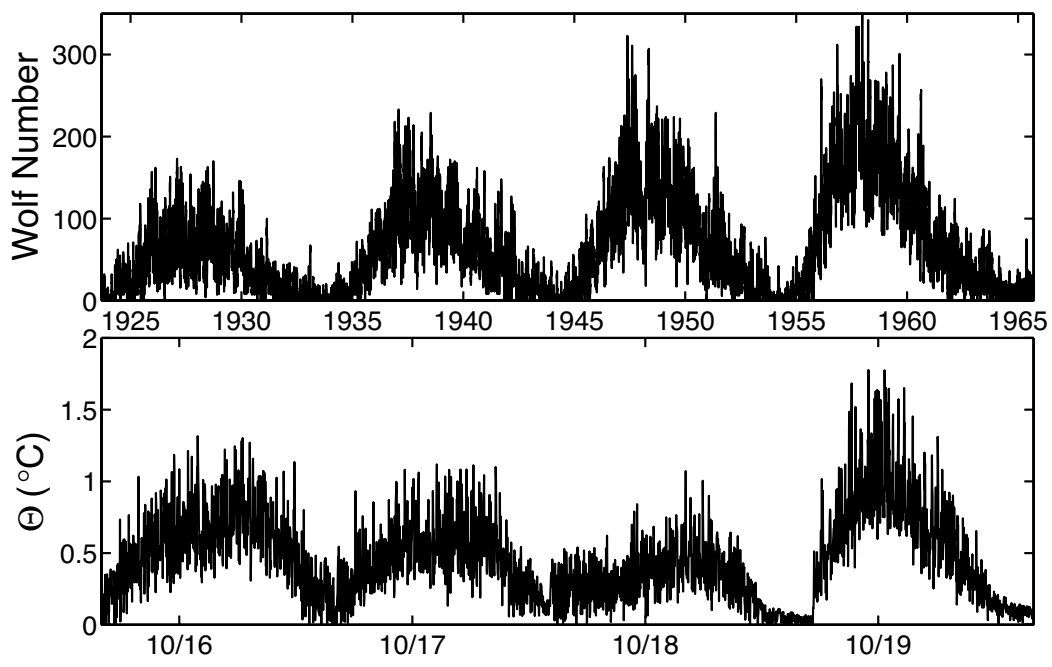

FIG. 9. Time series of Wolf number from 1924 to 1966 with a sampling time of one day, compared with a time series of $\Theta$ with a sampling time of 2 min measured during four days of October 2004.

time [5,7]. Recall that the Wolf number is defined as $s+10 g$ where $s$ and $g$ are the spot and spot group numbers, respectively. In the case of sunspots, the modulation has the dominating $11 \mathrm{yr}$ period of the intensity of the solar magnetic field, while in the case of the air avalanches, it is a diurnal modulation. The similarity between the temperature time series in the quarry pit and the sunspot Wolf number is actually striking in Fig. 9. In both cases, modulated Markov jitter offers a simple and adequate representation. The Sun and the access pit are undoubtedly two different physical systems; however, an analogy between the air avalanches and the sunspots may be worth contemplating. An interpretation of sunspots generated by plumes dragging the toroidal magnetic field up to the Sun's surface might justify this analogy [15-17]. The analysis presented in this paper could be applied to other time series and reveal that other natural phenomena can be modeled by a modulated Markov jitter.

\section{ACKNOWLEDGMENTS}

The authors thank the Inspection Générale des Carrières and the City of Paris for the access to the Vincennes quarry. Patrick Richon and Pierre Morat are also thanked for their contributions to the experiments. This is IPGP contribution number 2164.
[1] L. P. Kadanoff, Phys. Today 54(8), 34 (2001).

[2] K. R. Sreenivasan, A. Bershadskii, and J. J. Niemela, Phys. Rev. E 65, 056306 (2002).

[3] H.-D. Xi, S. Lam, and K.-Q. Xia, J. Fluid Mech. 503, 47 (2004).

[4] B. I. Shraiman and E. D. Siggia, Nature (London) 405, 639 (2000).

[5] E. M. Blanter, M. G. Shnirman, and J.-L. Le Mouël, J. Atmos. Sol.-Terr. Phys. 67, 521 (2005).

[6] M. Iosifescu, Finite Markov Processes and Their Applications (John Wiley and Sons, Chichester, 1980).

[7] E. M. Blanter, J.-L. Le Mouël, F. Perrier, and M. G. Shnirman, Sol. Phys. (to be published).

[8] F. Perrier, J.-L. Le Mouël, V. Kossobokov, C. Crouzeix, P. Morat, and P. Richon, Eur. Phys. J. B 46, 563 (2005).
[9] X.-L. Qiu and P. Tong, Phys. Rev. E 66, 026308:1 (2002).

[10] S. I. Vainshtein and K. R. Sreenivasan, Phys. Rev. Lett. 73, 3085 (1994).

[11] S.-Q. Zhou and K.-Q. Xia, Phys. Rev. Lett. 89, 184502:1 (2002).

[12] C. Crouzeix, Ph.D. thesis, Institut de Physique du Globe de Paris, 2005 (unpublished).

[13] F. Perrier, P. Morat, and J.-L. Le Mouël, Phys. Rev. Lett. 89, 134501:1 (2002).

[14] F. Perrier, P. Richon, C. Crouzeix, P. Morat, and J.-L. Le Mouël, J. Environ. Radioact. 71, 17 (2004).

[15] S. K. Solanki, Astron. Geophys. 43, 5.09 (2002).

[16] S. K. Solanki, M. Schüssler, and M. Fligge, Nature (London) 408, 445 (2000).

[17] E. Friis-Christensen and K. Lassen, Science 254, 698 (1991). 